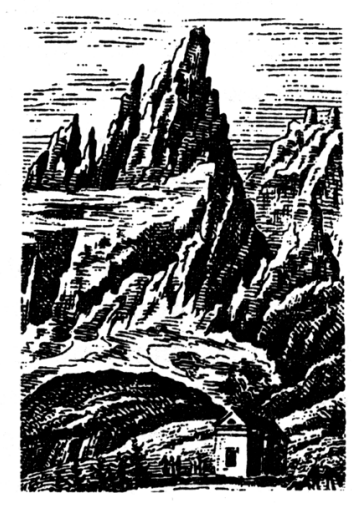

Sven Ismer

Philipps-Universität Marburg

sven.ismer@staff.uni-marburg.de

Nina Peter

Universität Bern

nina.peter@germ.unibe.ch

DOI: 10.19195/2084-4107.11.25

„Das alles ist Teil einer Reise zu sich selbst"* — Grenzerfahrungen als identitätsstiftendes Moment in zeitgenössischen Autobiographien von Bergsteiger*innen/

* Lynn Hill über das Klettern in einer großen Steilwand, Hill \& Child 2007, 123. 


\title{
„To wszystko jest częścią podróży do samego siebie" — doświadczenia graniczne jako moment kształtujący tożsamość we współczesnych autobiografiach wspinaczy
}

Słowa-klucze: Góry, alpinizm, wspinaczka sportowa, literatura górska

Keywords: climbing, liminal experience, autobiography

\section{"It's all part of the journey to yourself": Liminal experiences as identity-creating moments in contemporary climbers' autobiographies}

\author{
Summary
}

Experiencing physical and mental boundaries has always been part of mountaineering. However, over the last 150 years we have witnessed a process in which, in climbers' accounts, mountaineering and climbing become more and more important as liminal experiences. While in the so-called "golden age" of mountaineering (1850-1865) the authors focused on the first ascents of well-known summits and during the "heroic mountaineering" stage (1930s) they described primarily traverses of increasingly difficult routes, what comes to the fore in contemporary autobiographical works of professional climbers is the representation of subjective and individual liminal experiences. In recent autobiographies climbing gains importance as an individual quest for experiences and is presented as a form of self-fulfilment: liminal experiences of climbers become moments shaping their identity. The process is reflected in the style of climbing, which has evolved from collective expeditions to radical solo climbs. Speed or free solo climbs are an example of such an individualistic approach, in which grappling with oneself gets at least as much attention as grappling with the mountain. The authors of the article explore, from the perspective of literary studies and sociology, the representation of liminal experiences as identity-shaping moments in contemporary autobiographical works by Lynn Hill (Climbing Free, 2002), Catherine Destivelle (Ascensions, 2003), Alex Honnold (Alone on the Wall, 2015) and Andy Kirkpatrick (Psychovertical, 2008).

Lynn Hill, eine der besten Kletterinnen in der Geschichte des Alpinismus, spricht im Titel-Zitat von ihren Erfahrungen bei den oft tagelangen Besteigungen der weltberühmten Steilwände im amerikanischen Yosemite-Nationalpark. Die Konfrontation mit extremen Anstrengungen und Gefahren erscheint im Zitat als Weg zum Herausbilden von Identität, ein Zusammenhang, der seit vielen Jahren auch in den Sozialwissenschaften und der Psychologie diskutiert wird. Die strukturelle Bedeutung von Grenzerfahrungen wird dabei im ,Empfinden bzw. ,Spüren“ von Identität gesehen: „Leiblich-körperliche Grenzerfahrungen sind Erfahrungen, in denen das Individuum seine Leiblichkeit als innerer [sic] Widerstand spürbar realisiert [...]. Für die Identität sind leiblich-körperliche Grenzerfahrungen von Bedeutung, insofern sie zu Reflexionen Anlass geben und 
zu neuem Körperwissen sowie gegebenenfalls zur Modifikation von Körperpraktiken führen.“" (Gugutzer 2002, 277f.)

Dieser Beitrag rekonstruiert den Zusammenhang von körperlich-leiblichen Grenzerfahrungen und Identität im Bergsport anhand autobiographischer Texte von Kletterer*innen empirisch. Ausgewählt wurden dafür vier Texte von Athlet*innen, die in den letzten dreißig Jahren eine wichtige Rolle im Bereich des Kletterns gespielt haben. Die US-Amerikanerin Lynn Hill ist der erste Mensch, der die weltbekannte Route „The Nose“ am El Capitan (USA) frei (also ohne technische Hilfsmittel) kletterte - eine Leistung, die mehrere Jahre trotz zahlreicher Versuche nicht wiederholt werden konnte. Ihre Autobiographie Climbing Free erschien im englischsprachigen Original 2002. Die Französin Catherine Destivelle ist zunächst vor allem im Bereich des Wettkampfkletterns bekannt geworden, bevor sie sich dem Bergsteigen zuwandte und unter anderem als erste Frau die Eiger-Nordwand allein im Winter bestieg. 2003 veröffentlichte sie eine Autobiographie unter dem französischen Titel Ascensions. Andy Kirkpatrick ist ein britischer Kletterer, der sich besonders mit schwierigen Projekten im Bereich des technischen Kletterns einen Namen machte. Seine 2008 erschienene Autobiographie Psychovertical thematisiert ausführlich die Lese- und Rechtschreibstörung des Kletterers, der im Klettern ein im Alltag häufig unerreichtes Kompetenzerleben findet. Der vierte untersuchte autobiographische Text ist das 2015 unter dem Titel Alone on the Wall erschienene Buch von Alex Honnold (und seinem Ko-Autor David Roberts). Honnold ist der gegenwärtig vielleicht spektakulärste Kletterer, der in erster Linie durch ungesicherte free solo Besteigungen extremer Routen bekannt wurde.

Das Potential der Vertikalsportart Klettern, Grenzerfahrungen bei den Aktiven hervorzurufen, liegt auf der Hand. Mancherorts wird der Sport wegen seines symbolischen Spiels mit der Möglichkeit des Todes bereits als eine Art Antithese zur mit der Moderne einhergehenden Rationalisierung betrachtet (Lewis 2000). Das identitätsprägende Potential von Extremsportarten im Allgemeinen und dem Klettern und Bergsteigen im Besonderen hat bereits vielfach die Aufmerksamkeit psychologischer (z.B. Aufmuth 1983; Csikzentmihalyi \& Jackson 2000; Zuckerman 2007), aber auch soziologischer Forschung erregt. In den soziologischen Untersuchungen wird insbesondere der Zusammenhang zwischen dem sogenannten Extremsport und Identitätsproblematiken in (post-)modernen Gesellschaftsstrukturen (z.B. Bette 2004; Rummelt 2003) sowie die Rolle von Extrem- und Trendsportarten für die Konstruktion von Genderidentitäten fokussiert (z.B. Wheaton 2000; Thorpe 2005). Stets ist dabei auch die Frage nach den Beweggründen für das Eingehen des oftmals beträchtlichen Risikos Teil der Analysen. Dabei stellt ein weit verbreiteter Ansatz die Gefahr bzw. das mit der Kontrolle des scheinbar Unkontrollierbaren einhergehende Selbstwirksamkeitserlebnis in den Mittelpunkt der Erklärung (Lyng 1990; Reith 2005). Dieser sogenannten Edgework-These zufolge werden zwangsläufig immer größere Risiken eingegangen, um die damit einhergehenden Erlebnisse weiter zu steigern. Dem gegenüber stehen Studien, die eher den autotelischen Charakter der Flow-Erfahrung im Zentrum der Anziehungskraft und Popularität des Risikosports sehen (z.B. Hardie-Bick \& Bonner 
2013; Csikzentmihalyi \& Jackson 2000). Folgt man diesen Autorinnen, dann ist zwar die Bewältigung einer Herausforderung ein konstituierendes Merkmal der gesuchten Erfahrung, diese muss aber keineswegs mit einer Gefahr für Leib und Leben verbunden sein.

Wenn, wie in diesem Beitrag der Fall, der Blick vor allem auf die Rolle von Grenzerfahrungen gerichtet wird, sollte deshalb voran gestellt werden, dass diese vielerlei Gestalt annehmen können - nicht zuletzt, weil es aus Sicht des Individuums eine Vielzahl von Grenzen unterschiedlichster Natur gibt, denen in entsprechenden Erfahrungen begegnet werden kann, seien dies körperliche, mentale, ästhetische oder auch spirituelle. Grenzerfahrungen sind zunächst einmal Begegnungen mit dem Außeralltäglichen, was deren überaus subjektive Natur verdeutlicht. Sie gehen häufig mit einer Krise des Subjekts einher, einer Situation also, die mit Oevermann (2004) als Versagen eingeschliffener Routinen beschrieben werden kann. Das Individuum ist durch das Fehlen angemessener Verhaltensmuster gezwungen, neue Wege zu beschreiten, Routinen zu reflektieren und, falls möglich, zu modifizieren. Hier wird schon der große Stellenwert von Grenzerfahrungen für die „Identitätsarbeit“ (Keupp et al. 1999) des Individuums in der Moderne bzw. Postmoderne deutlich. Für Subjekte der Gegenwart ist Identität in den seltensten Fällen primordial erlangte Gewissheit, sie ist vielmehr eine lebenslange Aufgabe. In dieser Hinsicht sind krisenhafte Grenzerfahrungen nicht zwangsläufig etwas Negatives, bieten sie doch immer auch Chancen der Erweiterung, des Wachsens und nicht zuletzt auch der Distinktion in einer sozialen Welt, die vom Individuum verlangt, etwas Besonderes zu sein.

Betrachtet man die Geschichte des Alpinismus anhand autobiographischer Texte ihrer Protagonisten und Protagonistinnen, dann lässt sich in dieser Geschichte ein Trend zu einer Fokussierung von individuellen Auseinandersetzungen mit immer neuen Grenzen feststellen. Während im sogenannten ,goldenen Zeitalter ${ }^{6}$ des Alpinismus (1850-1865) Erstbesteigungen berühmter Gipfel im Dienste einer wissenschaftlichen Auseinandersetzung im Fokus alpinistischer Aktivitäten standen, und in der Phase des sogenannten ,heroischen Alpinismus ${ }^{6}$ in der ersten Hälfte des 20. Jahrhunderts in erster Linie die Bezwingung immer schwierigerer Routen, häufig im Namen des Ruhmes einer Nation, zu verzeichnen war (zur Geschichte des Alpinismus vgl. u.a. Grupp 2008), rücken in aktuellen autobiographischen Texten von professionellen Bersteiger*innen und Kletterer*innen neben der Beschreibung äußerer Erfolge Darstellungen subjektiver und individueller Grenzerfahrungen in den Mittelpunkt. Bergsteigen und Klettern gewinnt mittlerweile einen Großteil seiner Bedeutung aus seinem Potential für eben solche individuellen Grenzerfahrungen und wird als Form der Selbstverwirklichung und -findung betrachtet und inszeniert. Mit dieser Entwicklung geht ein Begehungsstil einher, der sich von gemeinschaftlichen Expeditionen hin zu radikalen Soloaktionen entwickelt hat. Speed- oder Free-Solo-Begehungen sind Beispiele dieser individualistischen Herangehensweise, die der Bezwingung oder 
auch Befreiung des Selbst eine mindestens so große Aufmerksamkeit widmet wie der Bezwingung des Berges.

Für die Auseinandersetzung mit dem stets problematischen Thema „Identität“ in Autobiographien bietet es sich an, auf das Konzept der „narrativen Identität" (Ricœur 1987) zurückzugreifen. Identität entsteht wesentlich, so die Annahme dieser Herangehensweise, durch eine narrative Strukturierung - ein emplotment - vergangener Erfahrungen und Erlebnisse, wodurch diese zu einer kohärenten Geschichte zusammengefügt werden, die in der Rückschau Sinn konstruiert und attribuiert. Beschäftigt man sich also mit Autobiographien — der Narration des eigenen Lebens - so können diese hinsichtlich ihrer narrativen Verfasstheit (beispielsweise ihrer Chronologie, ihrer Erzählerfiguren, ihrer Erzählund Gattungsmodelle oder ihrer selbstbezüglichen Aussagen) befragt werden (vgl. Lucius-Hoene \& Deppermann 2004). Diese Perspektive ist mit Implikationen in Bezug auf die Analyse und ihre Ergebnisse verbunden: Die Texte werden nicht als Repräsentation von Realität oder als Abbild dessen, was war, analysiert, sondern als Handlung im Hier und Jetzt des autobiographischen Schreibens, welche sich interaktiv an ein Publikum richtet (vgl. Schäfer \& Mayer 2011, 82).

Viele der unzähligen zeitgenössischen autobiographischen Kletter- und Alpin-Berichte rücken das Moment der Erfahrung der Gefahr und des Risikos in den Fokus. Lynn Hill und Catherine Destivelle - zwei Frauen, die in den vergangenen 30 Jahren die Geschichte des Kletterns entscheidend geprägt haben — beginnen ihre Autobiographie beide mit der Erzählung eines Sturzes, den sie jeweils nur mit viel Glück überlebt haben. Beide Autorinnen brechen mit dem Erzählen des Unfalls aus der Chronologie des Berichts ihrer Lebensgeschichte aus und stellen eine einschneidende Grenzerfahrung an den Beginn. In beiden Texten leitet der Sturz die Reflexion des Kletterns und der eigenen Sportlerinnen-Biographie ein. Die Autorinnen erzählen vom Sturz her, aber zugleich auch ,gegen' den Sturz an, indem sie der Frage nachgehen, wie sich ihr Klettern zu dem erwiesenermaßen hohen Risiko verhält, das sie damit immer wieder eingehen, und welcher Gewinn das Eingehen der Gefahr rechtfertigt und legitimiert: „Was bedeutete dieser Sturz? Was war mein Schicksal und der Sinn in meinem Leben? Das Klettern hatte mein Leben bestimmt, es lag mir im Blut, aber wenn man von mir wissen wollte, warum ich kletterte, mußte ich bei der Erklärung nach Worten suchen.“ (Hill \& Child 2007, 26)

Für Hill, wie auch für Destivelle, dient die „Begegnung mit dem Tod“ (ebd., 27) als Anlass, ihr Klettern und ihre Kletterphilosophie zu reflektieren und ihren Werdegang als Kletterin zu erzählen. In der Reflexion von Hill wird dabei deutlich, dass es keineswegs die Gefahr ist, die das Klettern für sie zum zentralen und identitätsstiftenden Bestandteil ihres Lebens macht. Sie ist zwar immer wieder bereit, großes Risiko einzugehen, sieht den ,Sinn' (ebd., 57f.) ihres Kletterns aber nicht in der Grenzerfahrung der Lebensgefahr: „Ich brauchte keinen Flirt mit dem Tod. Ich war zufrieden mit der Herausforderung, das Freiklettern als Mittel zu nutzen, Frieden und Harmonie in meinem Leben zu finden.“ (Ebd., 
304) Mit dieser Perspektive auf das Klettern steht Hill für eine große Mehrheit der Szene, wie eine Studie von Hardie-Bick und Bonner (2016) zeigen konnte. Entgegen der lange die Forschung zu Extrem- und Risikosportarten dominierenden Edgework-These, derzufolge das Risiko (und das Erleben der Kontrolle des eigentlich Unkontrollierbaren) im Mittelpunkt dieser Aktivitäten steht und stetig gesteigert werden muss, zeigen die Ergebnisse von Hardie-Bick und Bonner, dass eine große Mehrheit der Aktiven eher nach Möglichkeiten der Reduzierung von Risiken als nach deren Steigerung sucht; Adrenalin wird keineswegs gesucht, sondern erscheint wegen der mit einem Adrenalinerleben einhergehenden Gefahr des Kontrollverlusts sogar als „Feind“ der Kletterer*innen (ebd., 379).

Für Lynn Hill geht es beim Freiklettern am Fels darum, „die eigenen Fähigkeiten den natürlichen Eigenschaften des Gesteins anzupassen“ (Hill \& Child 2007, 262f.). Die Lösung des „Puzzles“ (ebd., 50), das der Fels ihrem Körper aufgibt, erlebt sie auch unabhängig vom Risiko, das sie dabei eingeht, als intensive Selbsterfahrung. Die eigentliche Motivation ihres Kletterns ist also nicht der Nervenkitzel der Lebensgefahr, sondern eine andere Form der Grenzerfahrung, die sich als ,Flow'-Erlebnis im Kontakt mit der natürlichen Umwelt beschreiben lässt: „Für mich ist das Klettern ein Mittel, um einen Zustand reinen Bewußtseins zu erlangen, bei dem es keine Ablenkungen oder Erwartungen gibt. Dieser intuitive Zustand des Seins erlaubt es mir, Augenblicke der wahren Freiheit und Harmonie zu erleben.“ (Ebd., 310) Mit dem Begriff des ,Flow' bezeichnet der Psychologe Csíkszentmihályi einen psychologischen Zustand, der durch ein restloses Aufgehen in einer Tätigkeit und ein hohes Maß an Konzentration und Vertiefung charakterisiert ist (Csíkszentmihályi 2010). Im Flow werden fordernde Aufgaben scheinbar mühelos bewältigt, Handeln und Bewusstsein verschmelzen miteinander, das Zeitgefühl verändert sich und die Tätigkeit wird unmittelbar als befriedigend und bereichernd erfahren. Fast paradigmatisch finden sich die Charakteristika dieser außeralltäglichen Erfahrungsweise in Hills folgender Beschreibung eines ihrer ersten Kletterwege:

Jede Position meines Körpers zu kontrollieren und gleichzeitig jeden Gedanken an einen Sturz zu verbannen, hatte eine interessante Wirkung: das Gefühl, dass mir alles und nichts im selben Augenblick bewusst war. Alles, weil ich mich 25 Jahre später immer noch an das Kaleidoskop der Kristallmuster im Gestein vor mir erinnere, und nichts, weil ich in dem Moment so vertieft in die Bewegung war, dass ich weder Zeit, Schwerkraft noch meine eigene Existenz spürte. Das einzige, was ich wahrnahm, war mein Atem (Hill \& Child 2007, 50).

Auffallend häufig finden sich Beschreibungen von Flow-Erfahrungen bei Lynn Hill jedoch, wenn sie von Situationen berichtet, in denen sie ein Risiko eingeht: „Ich hatte keine Ahnung, welchen Schwierigkeitsgrad ich gerade geklettert war, allerdings wusste ich, dass der Abschnitt sehr hart und potentiell lebensgefährlich gewesen war. Ich hatte mich so stark konzentriert, dass ich alles 
um mich herum vergessen hatte [...]. [A]lles schien in diesem Augenblick heller zu leuchten“ (ebd., 189). Die Lebensgefahr wird nicht als Selbstzweck aufgesucht, sondern erscheint als bisweilen unvermeidlicher Teil der Art des Kletterns, für die sich Hill begeistert, als Teil des „Pakt[s], den man mit dem Fels geschlossen hatte" (ebd., 186). Zugleich scheint die Lebensgefahr das Zustandekommen einer Flow-Erfahrung zu begünstigen: In gefährlichen Situationen macht gerade die Notwendigkeit, die Gefahr auszublenden, um handlungsfähig zu bleiben, das Zustandekommen der erhöhten Konzentration und der Vertiefung in die eigenen Bewegungen wahrscheinlicher (vgl. ebd., 50f.). Eine ähnliche Beobachtung lässt sich auch bei Hills größter Wettkampf-Konkurrentin Catherine Destivelle machen. Diese beschreibt das besonders riskante Solo-Klettern, dem sie sich nach dem Ende ihrer Wettkampfkarriere zuwendet, als einzigen Weg zur ,perfect osmosis with the rock“, es ermögliche einen ,instinctive almost animal way of climbing“" (Destivelle 2015, 88). Diese Erfahrung stellt sie als ihren Antrieb dar. Sie lässt sich als eine Transzendierung körperlicher Grenzen beschreiben, die für Destivelle nur in den Bergen und nur beim Soloklettern erfahrbar ist und ihr höchste Befriedigung verschafft.

Dass die Lebensgefahr grade durch die Notwendigkeit ihrer Ausblendung eine als genussvoll erlebbare Fokussierung auf das Hier und Jetzt ermöglicht und zu einem Zustand des Einklangs mit dem eigenen Körper und seiner Bewegung in der Umgebung führen kann, wird besonders bei der Lektüre der Autobiographie von Alexander Honnold deutlich. Honnold ist bekannt für seine spektakulären ungesicherten Free-Solo-Begehungen schwieriger und langer Kletterrouten. Für Honnold ist jedoch sein Kletterstil in erster Linie ein Mittel, höchste Leistungen und höchste Konzentration zu erreichen, das Risiko ist kein Ziel an sich: „Ich liebe die Einfachheit des Solo-Kletterns. Du kletterst nie besser als solo.“ (Honnold \& Roberts 2016, 87); „Wenn ich free solo klettere, bin ich total auf das konzentriert, was vor mir ist, auch in den leichteren Seillängen. Das ganze Universum schrumpft auf mich und den Fels zusammen." (Ebd., 55) Die wesentliche Bedingung für das Entstehen dieses Zustandes erhöhter Konzentration ist eine paradoxe Gleichzeitigkeit des Wissens und Nicht-Wissens um das Risiko: „Grundsätzlich habe ich, wenn ich solo klettere, normalerweise so etwas wie einen mentalen Panzer. Man könnte sagen, ich bin in einem Zustand höchster Konzentration. Etwas schützt meinen Kopf davor, zu viel zu denken.“ (Ebd., 87)

Für seine Autobiographie wählt Honnold eine Erzählstrategie, die diese Gleichzeitigkeit von Bewusstsein und Ausblendung der Gefahr auf einer narrativen Ebene spiegelt: Honnolds (Auto)Biographie ist zweistimmig erzählt: Textpassagen von Honnold selbst wechseln ab mit Textpassagen, in denen sein Ko-Autor David Roberts über Honnold schreibt. In dieser Außenperspektive wird immer wieder die Lebensgefahr thematisiert, in die sich Honnold begibt: „Was Alex fertigbrachte, war, weit schwierigere und auch längere Routen free solo zu begehen, als irgendjemand vor ihm je für möglich gehalten hätte. Bis jetzt ist er heil davongekommen, auch wenn einige seiner besten Freunde befürchten, dass er dabei 
umkommen wird.“ (Ebd., 15) Der Ko-Autor liefert die Beschreibung des Risikos, das Honnold eingeht, während Honnold selbst die Relevanz dieses Risikos für seine Klettermotivation immer wieder relativiert: „Ich mag das Risiko nicht. [...] Deshalb versuche ich, mein Solo-Klettern möglichst sicher zu machen, sodass es unwahrscheinlich ist, dass ich abstürze, auch wenn es wirklich schlimme Folgen hätte, wenn ich es täte.“ (Ebd., 19) Die von Honnold gesuchte Klettererfahrung ist also nicht das Risikoerlebnis selbst oder allein, sondern der Flow am Fels, der paradoxerweise gerade in Risikosituationen dadurch zustande kommt, dass erwartbare Emotionen wie Angst und Nervosität nicht empfunden werden: „Schon bald war ich soweit, dass ich mich beim Solo-Klettern recht wohlfühlte. Ich machte eine Entdeckung: Wenn ich eine besondere Stärke hatte, dann war es eine mentale - die Fähigkeit, die Ruhe auch in Situationen zu bewahren, in denen man normalerweise nervös wird.“ (Ebd., 25) Die Kombination aus Honnolds Fähigkeit, Angst und Nervosität auszublenden mit dem stilistischen Mittel der Ko-Narration von Roberts, ermöglicht es den Rezipient*innen seiner Filme und Texte umgekehrt, genau diese Emotionen zu durchleben und Honnold gewissermaßen als Medium einer empathischen Grenzerfahrung — der imaginierten Lebensgefahr und des vorgestellten Eingehens eines hohen Risikos — zu nutzen. Genau diese passive Grenzerfahrung, das empathische Erlebnis der Rezipient*innen, wird im Text immer wieder thematisiert und beschrieben. Roberts berichtet ausführlich über die Reaktionen auf Honnolds Klettern, bei denen in der Regel das Moment der Lebensgefahr und die Frage nach seiner Motivation im Zentrum steht: „Immer und immer wieder werden ihm bei seinen öffentlichen Auftritten dieselben Fragen gestellt [...]. Es sind diese grundlegenden Fragen zu dem, was er am Fels treibt: Haben Sie keine Angst, dabei umzukommen? Warum machen Sie das?" (ebd., 16) Auffallend häufig beschreibt Roberts, was Zuschauer*innen der Filmaufnahmen von Honnolds Begehungen während der Betrachtung durchleben: „Niemand kann ihn ansehen - selbst wenn es zum vierten oder fünften Mal ist —, ohne dass seine Handflächen schweißnass werden [...]. Manche Nichtkletterer bringen es gar nicht über sich, den Film anzuschauen." (Ebd., 85) Besonders deutlich wird die Wirkung der Betrachtung Honnolds in einem in die Biographie eingefügten Zitat eines Kameramanns, der sich ganz buchstäblich mittels der Kameraeinstellung vom kletternden Honnold distanzieren muss, weil die im Betrachten erlebte Grenzerfahrung ihn überfordert: „Ich war entsetzt. Es war der beängstigendste Moment meines Lebens. Ich hielt es nicht mehr aus, ihm aus solcher Nähe zuzuschauen, ich musste den Zoom zurückfahren.“ (Ebd., 160) Schließlich beschreibt sogar Honnold selbst den Effekt seiner eigenen Filme in der Diskrepanz zur Klettererfahrung als „beängstigend“ und gesteht ein, dass er selbst beim Betrachten seiner Filme feuchte Hände bekommt (ebd., 277): „Das Seltsame ist, dass es schwerer fällt, beim Free-Solo-Klettern zuzuschauen, als es selbst zu tun.“ (Ebd.)

Für die Kletterer*innen selbst steht also laut den autobiographischen Beschreibungen als entscheidende Erfahrung der Flow und der Einklang mit dem 
Fels im Mittelpunkt ihres Kletterns - eine Erfahrung, die allerdings deutlich schwieriger zu narrativieren und zu spektakularisieren ist, als die objektive Gefahr, die der zentrale Fokus der Rezipient*innen ihrer Berichte und Filme zu sein scheint. Sowohl Lynn Hill als auch Alex Honnold beklagen eine Fokussierung der Medien auf besonders riskante Aktionen, während ebenso bemerkenswerte, aber weniger spektakuläre Leistungen weniger Aufmerksamkeit erfahren (vgl. z.B. Hill \& Child 2007, 340).

Das Klettern und die mit ihm verbundenen Grenzerfahrungen sind für die hier thematisierten Kletterer*innen nicht nur Lebensinhalt, sondern auch Quelle ihrer Selbsterfahrung und ihres Selbstbildes. Indem sie von ihren Klettererfahrungen, ihrer Beziehung zum Klettern sowie ihrer Kletterphilosphie und -ethik erzählen, entwerfen sie narrativ eine Vorstellung von sich selbst. Nicht nur die durch das Klettern bestimmte unkonventionelle, freiheitliche Lebensweise (vgl. z.B. Honnold \& Roberts 2016, 52, 93) und ihre ,Identifikation“ mit der „Gemeinschaft der Kletterer“, die zur Ersatzfamilie wird (ebd., 296; Hill \& Child 2007, 65), bestimmen ihre Identität - sie erfahren vielmehr auch und besonders einzelne Momente konkreter Routen als , sinnhaft' und als Bestandteil einer identitätsstiftenden Selbsterfahrung: „Ich erkannte den Sinn des Bigwall-Kletterns. Die Wand war wie ein Lebewesen, das unser Stehvermögen testete, indem es uns immer wieder mit Schwierigkeiten und neuen unbekannten Abschnitten konfrontierte, die wir überwinden mußten. [...] [D] as alles ist Teil einer Reise zu sich selbst und zu größerer Selbständigkeit.“ (Ebd., 123) Für Hill ist das Klettern ein Bereich, in dem sie Fähigkeiten unter Beweis stellen und weiterentwickeln kann, die sie für zentrale Teile ihrer Persönlichkeit hält. Zugleich wird für sie beim Klettern und besonders in Momenten der Grenzerfahrung ein intuitiver Umgang mit der Umgebung möglich, in dem sie sich als authentisch und „mit meiner Umgebung im Einklang“ (Ebd., 201) erlebt: „In solchen Situationen achtete ich genau auf meine innere Stimme, die am Boden so oft von den Geräuschen und Ansprüchen des Alltags übertönt wird.“ (Ebd., 142) Folgerichtig empfindet sie ihr Klettern als „Demonstration der Werte, von denen ich überzeugt war" (ebd., 280). Die Beschreibung der mentalen Vorgänge vor der Bewältigung der Schlüsselstelle einer Route zeigen, dass Hill Energie für die Bewältigung der schwierigen Passagen gewinnt, indem sie sich unmittelbar vor ihrem Kletterversuch ,an die Werte [erinnert], die mir im Leben und beim Klettern stets wichtig waren." (ebd., 267) Den gelingenden Kletterversuch versteht sie dann (zirkulär) wiederum als Ausdruck und Demonstration dieser Werte: „Für mich war dieser Versuch am Great Roof die Gelegenheit, die Stärke des freien Geistes und Denkens zu zeigen“ (Ebd., 267). Auch Honnold empfindet sein Klettern als Ausdruck seiner Werte und seiner Lebensphilosophie: „Ein Speed-Rekord an einem Bigwall ist die ultimative Bestätigung, dass ich so effizient geklettert bin, wie ich kann. Das steht im Einklang mit meiner Lebensphilosophie, meinem Fokus auf die Einfachheit, meinem bewusstem Verzicht auf Unnötiges.“ (Honnold \& Roberts 2016, 144) Umgekehrt überträgt auch Honnold Prinzipien seines Kletterns auf andere Lebensbereiche: 
„,ich glaube, wenn man sein Können am Fels [...] perfektioniert, verbessert man sich auch in anderen Bereichen. Ich bin davon überzeugt, dass das, was ich vom Klettern gelernt habe, sich auch auf andere Aspekte des Lebens übertragen hat.“ (Ebd., 287); „Ich versuche an den Umweltschutz so heranzugehen wie an die Felsen“ (ebd., 206). Für Hill und Honnold ist Klettern Ausdruck ihrer Werte und ihrer Lebensweise, also aufs Engste mit dem (narrativ entwickelten) Selbstverständnis verknüpft. Umgekehrt beziehen sie aus dem Klettern Prinzipien und Handlungsweisen für andere Lebensbereiche, das Klettern wird zur Orientierungsgröße für das Handeln jenseits der Berge.

Auf der anderen Seite kann das Klettern aber auch zu einer erleichternden Kontrasterfahrung zur Alltagsrealität und zu einem bestätigenden Selbstwirksamkeitserlebnis jenseits alltäglicher Anforderungen werden. Als Beispiel für eine solche Erfahrung des Kletterns lässt sich der Auftakt der Autobiographie des Bergsteigers Andy Kirkpatrick lesen. Dem einleitenden Kapitel seines Textes kommt eine Schlüsselrolle bei der narrativen Konstruktion von Identität zu. Der schottische Bergsteiger verbindet in diesem Kapitel zwei zeitlich weit auseinanderliegende Situationen miteinander, springt nach jeweils ein oder zwei Absätzen zwischen ihnen hin und her. Einerseits erzählt er von einem Lerntest während seiner Schulzeit, bei dem er in fast allen Bereichen versagt; lediglich im Bereich des räumlichen Denkens bescheinigt ihm der Test überdurchschnittliche Fähigkeiten. Bei ihm wird eine Legasthenie diagnostiziert, eine Hälfte seines Hirns „funktioniert nicht so, wie sie sollte“ (Kirkpatrick 2015, 16). Insgesamt empfindet Kirkpatrick den Test als unangenehme und bedrohliche Situation: sein Gehirn verwandelt sich in „eine zähe Masse aus Klebstoff“ (ebd., 10), ihn überfällt eine „panische Angst“" (ebd.).

Abschnittweise verwoben ist die Erinnerung an diese Situation mit der Schilderung einer schwierigen Bergbesteigung im winterlichen Schottland, von der ihm zuvor prophezeit wurde, dass er sie unmöglich bewältigen könne, da ihm mit seiner eher kleinen Statur für die Schlüsselpassage die Reichweite fehle. Sein schon im Test festgestelltes räumliches Vorstellungsvermögen ermöglicht es ihm wider Erwarten, die extrem schwere Winter-Begehung des schottischen Berges zu meistern.

Mit Hilfe der Verschränkung beider Episoden durch eine anachronische Erzählweise macht der Autor klar, welchen Stellenwert das Klettern für ihn hat. Das Klettern ist der Bereich des Lebens, in dem Kirkpatrick sich kompetent fühlt und in dem er Selbstwirksamkeit erfährt. Es steht damit in großem Kontrast zu den Herausforderungen des Alltags, denen er sich häufig nicht gewachsen fühlt, da er immer wieder an vermeintlich unüberwindliche Grenzen stößt und als „,behindert" (ebd., 14) eingestuft wird. Sein häufig riskantes Klettern ermöglicht es Kirkpatrick, eigene Fähigkeiten in Situationen unter Beweis zu stellen, in denen sein Überleben von ihnen abhängt, ihnen also eine unmittelbar erfahrbare Relevanz zukommt. 
In der Gesamtschau lassen sich aus den untersuchten Texten eine Reihe von Thesen zum Verhältnis von Grenzerfahrungen und Identitätsnarration generieren. Eine zentrale Rolle spielen dabei zwei Typen von Grenzerfahrungen, die einerseits von unterschiedlicher Natur sind, dennoch aber in einem Abhängigkeitsverhältnis zueinander zu stehen scheinen. Zum einen ist dies die Erfahrung körperlicher Transzendenz über das Aufgehen im Flow des Kletterns am Fels. Dieser Erfahrung wird in allen untersuchten Texten zentrale Bedeutung zugesprochen, sie ist es, aus der die Bergsteiger*innen und Kletterer*innen ihre intensivsten Selbsterfahrungen generieren. In manchen Fällen wird sie begünstigt durch den Aspekt des Risikos für Leib und Leben, der insbesondere im Fall von Alex Honnold erst die totale Konzentration auf den Augenblick ermöglicht, die für das ,Eins-Werden“ mit dem Fels nötig ist. Hier ließe sich also auf die eingangs angeführte Disparität der Literatur, in der die Studien zum Edgework denjenigen zum Flow gegenüberstehen, entgegnen, dass sich in der Empirie auch Fälle finden lassen, in denen beides Hand in Hand geht.

Die mit den Flow-Erfahrungen verbunden en Anstrengungen und Herausforderungen lassen sich, wie besonders von Lynn Hill und Alex Honnold geschildert wird, durch eine Rückbesinnung auf die individuellen persönlichen Werte bewältigen, werden also als eine in den Autobiographien narrativ ausgestaltete Erfahrung der Wirksamkeit der individuellen Lebensphilosophie erlebt. Umgekehrt werden die teilweise extremen Unternehmungen und Erfolge als Ausdruck und Demonstration eben dieser persönlichen Werte gedeutet. Diese Grenzerfahrungen bilden somit ein Grundelement der untersuchten Identitätsnarrationen.

\section{Literatur}

Aufmuth, Ulrich (1983): Risikosport und Identitätsproblematik. In: Sportwissenschaft 13, 249-270. Bette, Karl-Heinrich (2004): X-treme: Zur Soziologie des Abenteuer- und Risikosports. Bielefeld:

Transcript.

Csíkzentmihályi, Mihály; Jackson, Susan (2000): Flow im Sport. München: BLV.

Csíkszentmihályi, Mihály (2010): Das Flow-Erlebnis. Jenseits von Angst und Langeweile - Im Tun aufgehen. Stuttgart: Klett-Cotta.

Destivelle, Catherine (2015): Rock Queen. South Stainmore: Hayloft.

Grupp, Peter (2008): Faszination Berg. Die Geschichte des Alpinismus. Köln: Böhlau.

Gugutzer, Robert (2002): Leib, Körper und Identität. Eine phänomenologisch-soziologische Untersuchung zur personalen Identität. Wiesbaden: Springer.

Gugutzer, Robert (2004): Trendsport im Schnittfeld von Körper, Selbst und Gesellschaft. In: Sport und Gesellschaft 1(3), 219-243.

Hardie-Bick, James; Bonner, Penny (2016): Experiencing Flow, Enjoyment and Risk in Skydiving and Climbing. In: Ethnography 17(3), 369-387.

Hill, Lynn; Child, Greg (2007): Climbing Free: In den steilsten Wänden der Welt. München: Piper. Honnold, Alexander; Roberts, David (2016): Allein in der Wand. München: Piper.

Keupp, Heiner; Ahbe, Thomas; Gmür, Wolfgang; Höfer, Renate; Mitzscherlich, Beate; Kraus, Wolfgang; Sraus, Florian (1999): Identitätskonstruktionen. Das Patchwork der Identitäten in der Spätmoderne. Reinbek bei Hamburg: Rowohlt. 
Kirkpatrick, Andy (2015): Psychovertikal: Wenn Klettern zum Leben wird. Zürich: AS Verlag.

Lewis, Neil (2000): The Climbing Body, Nature, and the Experience of Modernity. In: Body \& Society 6(3-4), 58-80.

Lucius-Hoene, Gabriele; Deppermann, Arnulf (2002): Rekonstruktion narrativer Identität. Ein Arbeitsbuch zur Analyse narrativer Interviews. Wiesbaden: Springer.

Lyng, Stephen (1990): Edgework: A Social Psychological Analysis of Voluntary Risk-Taking. In: American Journal of Sociology 95(4), 851-886.

Oevermann, Ulrich (2004): Sozialisation als Prozess der Krisenbewältigung. In: Geulen, Dieter; Veith, Hermann (Hrsg.): Sozialisationstheorie interdisziplinär. Aktuelle Perspektiven. Stuttgart: Lucius \& Lucius, 155-181.

Reith, Gerda (2005): On the Edge: Drugs and the Consumption of Risk in Late Modernity. In: Lyng, Stephen (Hrsg.): Edgework: The Sociology of Risk-Taking. New York: Routledge, 227-246.

Ricœur, Paul (1987): Narrative Identität. In: Heidelberger Jahrbücher XXXI, 57-68.

Rummelt, Peter (2003): Risikosport in der Risikogesellschaft. In: Gissel, Norbert; Schwier, Jürgen (Hrsg.): Abenteuer, Erlebnis und Wagnis. Hamburg: Czwalina, 203-220.

Schäfer, Christina; Mayer, Gerhard (2011): Grenzerfahrung, Krise, Identität: Biographische Integration außergewöhnlicher Erfahrungen. In: Zeitschrift für Anomalistik 11, 78-112.

Thorpe, Holly (2005): Jibbing the Gender Order: Females in the Snowboarding Culture. In: Sport in Society 8(1), 76-100.

Wheaton, Belinda (2000): New Lads? Masculinities and the New Sport Participant. In: Men and Masculinities 2(4), 434-456.

Zuckerman, Marvin (2007): Sensation Seeking and Risky Behavior. Washington: APA.

Góry, Literatura, Kultura 11, 2018

(C) for this edition by CNS 